\title{
Implementing the NHS England Long Term Plan for stroke: how should reperfusion services be configured? A modelling study
}

\author{
Michael Allen ${ }^{1}$, Kerry Pearn ${ }^{1}$, Gary A. Ford ${ }^{2}$, Phil White ${ }^{3}$, Anthony G. Rudd ${ }^{4}$, Peter McMeekin ${ }^{5}$, \\ Ken Stein ${ }^{1}$, Martin James ${ }^{1,6}$. \\ ${ }^{1}$ University of Exeter Medical School and the National Institute for Health Research (NIHR) \\ Applied Research Collaboration South West Peninsula (SWPenARC). ${ }^{2}$ Radcliffe Department of \\ Medicine, Oxford University and Oxford University Hospitals NHS Foundation Trust. \\ ${ }^{3}$ Translational \& Clinical Research Institute, Newcastle University and Newcastle upon Tyne \\ Hospitals NHS Foundation Trust. ${ }^{4}$ Kings College London and Guy's and St Thomas, NHS \\ Foundation Trust. ${ }^{5}$ Faculty of Health and Life Sciences, Northumbria University. ${ }^{6}$ Royal Devon and \\ Exeter NHS Foundation Trust.
}

\begin{abstract}
Objectives: To guide policy when planning reperfusion thrombolysis (IVT) and thrombectomy (MT) services for acute stroke in England, focussing on the choice between 'mothership' (direct conveyance to an MT centre) and 'drip-and-ship' (secondary transfer for MT after local IVT) provision and the impact of bypassing local acute stroke centres.
\end{abstract}

Methods: Computer modelling was used to estimate the likely outcomes from reperfusion therapies, along with admission numbers to units, based on expected times to IVT and MT.

Results: Without pre-hospital selection for LAO, 94\% of the population of England live in areas where the greatest clinical benefit accrues from direct conveyance to an IVT/MT centre. If this model was followed then net benefit from reperfusion is predicted to be increased from 31 to 34 additional disability-free outcomes / 1,000 admissions. However, this policy produces unsustainable admission numbers at these centres, and depletes all but 19 IVT-only units of all stroke admissions. Implementing a maximum permitted additional travel time to bypass an IVT-only unit, or using a pre-hospital test for LAO, both increase net benefit over the current drip-and-ship model, but produce a similar destabilising effect on acute systems of care. Use of IVT-only units manage admission numbers to IVT/MT centres.

Conclusions: The mothership model reduces time to MT at the cost of increased time to IVT, but the benefit of faster MT is predicted to lead to a modest improvement in overall outcomes.

Providing a sustainable national system of acute stroke care requires a hybrid of mothership and drip-and-ship provision. 


\section{Introduction}

In the UK, about 95,000 people are hospitalised each year with acute stroke [1,2], and over half of these people are left with long-term disability at great cost to individuals and society [3]. Disability and institutionalisation after ischaemic stroke are significantly reduced by reperfusion treatments intravenous thrombolysis (IVT) [4] and mechanical thrombectomy (MT) [5]. IVT is a treatment that may be considered for all potentially disabling ischaemic strokes (which are about $88 \%$ of all strokes [6]) presenting in a treatable time window, whereas MT may be considered for all large artery occlusions (LAO), which are present in about $40 \%$ of all acute ischaemic strokes [7].

In 2019-20 in the UK these treatments were given to $11.7 \%$ and $1.8 \%$ of patients with acute stroke respectively [2]. The 2019 NHS England Long Term Plan [3] set out the ambition that, by 2025, acute stroke units will deliver IVT to about $20 \%$ of patients, and MT to about $10 \%$ [8]. To achieve such increases in reperfusion, the NHS England Long Term Plan acknowledges the need for the centralisation of hyperacute stroke care into fewer well-equipped and staffed hospitals [9], noting that metropolitan areas that have recently centralised stroke care have achieved improved outcomes $[10,11]$.

Two principal models for the provision of reperfusion are generally considered: the so-called mothership, where regional centres (sometimes referred to as comprehensive stroke centres) provide IVT and MT for all eligible suspected stroke patients, with ambulances bypassing local acute services; and drip-and-ship, where IVT is provided at smaller local units (sometimes referred to as acute stroke centres), followed by secondary transfer of selected patients to regional centres for MT. Currently, drip-and-ship is the predominant and commissioned model of provision in the UK. Reconfiguring hyperacute stroke services to a more centralised mothership model could increase direct access to MT for the eligible minority, but at the expense of increased travel times and delayed IVT for many patients; it could also substantially increase admissions to the regional MT centres, many of whom would be ineligible for reperfusion. Modelling and simulation has been suggested as being particularly useful in planning of stroke care for being able to generalise outside of local clinical studies [12]. We therefore set out to examine the impact of the reperfusion ambitions in the NHS England Long Term Plan for stroke on population disability, and to model the optimal organisation of hyperacute stroke provision necessary to achieve these ambitions in a sustainable way.

\section{Method}

\section{Location of thrombolysis and thrombectomy centres}

The location of thrombolysis centres was taken as the 107 hyperacute stroke units in England providing thrombolysis in 2019 [2]. Within this number, the location of IVT/MT centres, in our base case model, was taken as the 24 neuroscience centres in England which are either providing or planning to provide MT [2].

\section{Admission numbers and travel times for patients}

Patients are located to one of 32,833 Lower Super Output Areas (LSOA) in England. Admissions for stroke per LSOA for three years 2015-2017 were obtained from NHS Hospital Episodes Statistics, including 242,874 patients coded with an emergency admission of ischaemic or haemorrhagic stroke (primary diagnosis ICD-10 I61, I63, I64). When predicting the number of MT 
procedures, we have assumed that the proportion of confirmed stroke patients eligible for MT in the UK is $10 \%$, as previously estimated [13].

Travel times were estimated from all LSOA to all hospitals using Microsoft MapPoint. In a 'drip and ship' model, where an inter-hospital transfer may be required for MT, we assumed a net delay in MT of 60 minutes + inter-hospital transfer time (obtained from MapPoint).

\section{Clinical outcome}

Clinical outcome from reperfusion is quantified in terms of a disability-free outcome three months after stroke (a modified Rankin Scale score of 0-1/6 describes either no or only minor non-disabling symptoms [14]). We have published the detailed method and code used to estimate outcomes [15]. This method is a development of the method of Holodinsky et al. [16], and incorporates the known decay of effectiveness of IVT [4] and MT [17] over time.

For those patients with the prospect of treatment we assume it takes 60 minutes from stroke onset to the ambulance leaving the scene. Time to IVT is therefore 60 minutes + ambulance travel time + door-to-needle time (D2N). D2N times for IVT in the model are set to a standardised 40 minutes across all hospitals. For patients who attend an IVT/MT centre as their first admitting hospital, time to MT is given by 60 minutes + ambulance travel time + door-to-puncture time (D2P), where D2P for MT in the model are set to a standardised 90 minutes across all hospitals.

If a patient first attends a unit which does not provide MT, the time to MT incurs a further net delay and added inter-hospital travel time. The net delay used may be shorter than the actual door-in to door-out time at the first admitting hospital, as some process steps have already been completed on arrival at the MT centre. Previously, in a large clinical trial it was shown that patients receiving MT after transfer were treated 110 minutes later than patients admitted directly, 35 minutes of which was attributable to inter-hospital travel time, suggesting a net delay of 75 minutes + travel time [18]. In our modelling we have anticipated some improvement over these historical results and have assumed a 60 minute net delay in addition to the inter-hospital transfer travel time.

\section{Pre-hospital selection}

In recent years several methods for ambulance paramedics to select patients with LAO as potential candidates for MT have been developed [19]. We have used the performance of one of the most widely studied, the RACE pre-hospital diagnostic for LAO [20], to assess the potential impact of selection of patients most likely to benefit from direct transport to an IVT/MT centre, bypassing any nearer IVT-only centre.

\section{Varying number and location of thrombectomy centres}

For selecting locations of IVT/MT centres (in addition to the current 24 neuroscience centres) we have used a genetic algorithm as previously described [21]. The algorithm optimises choice of stroke units based on maximising access to stroke units and on achieving the minimum guideline admissions to as many hospitals as possible, while also keeping the number of admissions to the largest unit to as low as possible. National guidelines recommend a minimum number of admissions to an acute stroke unit, providing thrombolysis, of 600 patients per year [22], coupled to the recommendation that travel time to hyperacute stroke care should be ideally 30 minutes or less, and no more than 60 minutes [23]. A further competing priority is the minimum number of MT procedures to be performed by any given unit to maintain institutional expertise. In order to 
maintain the procedural skills of interventional teams and yet have sufficient specialists to staff a rota, we have used a minimum threshold of $150 \mathrm{MT}$ procedures per year for an IVT/MT centre [24].

\section{Results}

\section{Benefit of IVT and MT - Drip and ship}

We estimated net clinical benefit of a drip-and-ship model of care, where all patients are assumed to travel to their closest acute stroke unit (providing at least IVT) first, and travel onwards to their nearest IVT/MT centre if necessary. If current IVT rates (which range from 4 to $22 \%$ [6]) are used then the clinical benefit without and with $24 / 7 \mathrm{MT}$ at 24 current neuroscience centres would be 10.9 and 18.0 additional disability-free outcomes / 1,000 admissions. If a uniform $20 \%$ IVT rate was achieved then clinical benefit without and with 24/7 MT at 24 neuroscience centres would be 18.9 and 31.1 additional disability-free outcomes / 1,000 admissions. Figure 1 shows the geographical variation in clinical benefit with these scenarios. First hospital admissions in this model range from 259 to 1,964 . If patients attend their closest unit first, $27 \%$ directly attend an IVT/MT centre first.

The rest of the analysis presented assumes uniform 20\% IVT and 10\% MT rates.

\section{Benefit of IVT and MT - Mothership}

If all patients travelled to their nearest IVT/MT centres and bypassed a more local IVT-only unit, the delay in IVT and the effect on clinical outcome is shown in figure 2 . The net clinical benefit would be an additional 33.5 disability-free outcomes / 1,000 admissions. The mothership model therefore gives a modest net improvement in outcome over drip-and-ship; though importantly individual regions may have a poorer or better predicted outcome with a mothership model (ranging from a reduction of 6.0, to an increase of 13.8 additional disability-free outcomes / 1,000 admissions). First hospital admissions in this model range from 1,228 to 6,183, with 22 out of 24 IVT/MT centres receiving more than 2,000 patients per year.

\section{Destination based on most likely good outcome}

Instead of all patients being conveyed to their closest acute stroke unit as in the drip-and-ship model, or all patients travelling directly to their nearest IVT/MT centre as in the mothership model, it is possible to make a patient-specific decision by using a prediction, in the absence of any diagnostic test for LAO, as to whether disability outcome is improved by bypassing a more local IVT-only unit and travelling further to directly attend an IVT/MT centre. Overall clinical benefit is predicted to increase to 33.6 additional disability-free outcomes / 1,000 admissions, a marginal improvement over an exclusive mothership model. Figure 3 shows the effect that this has on the catchment area of IVT-only units and IVT/MT centres. Compared to the drip-and-ship model, the travel time to the first admitting stroke unit is, on average, increased from 19 to 34 minutes, but travel time to an IVT/MT centre is reduced from 94 to 42 minutes. Such a strategy causes significant disruption to admission numbers. Compared with the drip-and-ship model, the proportion of patients directly attending an IVT/MT centre rises from $27 \%$ to $94 \%$, and average first admissions to an IVT/MT centre rises from 918 to 3,187, with the largest centre increasing from 1,964 to 5,578. Average first admissions to IVT-only units fall from 572 to 43, with 64 out of 83 IVT-only units depleted of all their stroke admissions. 


\section{Destination based on maximum additional travel time}

So far we have examined the effect of bypassing a nearer IVT-only centre in order to directly attend an IVT/MT centre for all patients (mothership model), and just for those for whom it is expected that bypass will confer a better clinical outcome. Alternatively we can assess the impact of allowing a maximum additional travel time in order for a patient to travel directly to an IVT/MT centre. In previous studies we have used the concept of ambulance bias, defined as the additional time that an ambulance crew is willing to travel in order to convey a patient directly to an IVT/MT centre. As ambulance bias increases, a number of effects are observed (figure 4): average time to IVT is increased, average time to MT is reduced, net clinical outcome is improved, admissions to IVT-only units fall, and admissions to IVT/MT centres increase. For example, if ambulance crews are willing to travel 15 minutes further in order to directly attend an IVT/MT centre, the net benefit of reperfusion treatment increases from 31.1 to 32.3 additional good outcomes per 1,000 admissions. However, the proportion of all stroke patients admitted directly to an IVT/MT centre would increase from $27 \%$ to $52 \%$. The number of units with fewer than 300 first admissions per year increases from three to 18 , while the number of units with fewer than 600 first admissions per year increases from 39 to 64 (out of 83 IVT-only units in total). At the same time, the number of units with more than 2,000 first admissions increase from zero to eight.

\section{Pre-hospital selection for large artery occlusion}

Applying the RACE pre-hospital diagnostic test for LVO, with all 'RACE-positive' patients taken directly to an IVT/MT centre, increases the number of additional disability-free outcomes from 31.1 to 33.4 per 1,000 admissions. However, the proportion of all stroke patients admitted directly to an IVT/MT centre would increase from $27 \%$ to $62 \%$. The number of units with fewer than 300 first admissions per year increases from three to 30, while the number of units with fewer than 600 first admissions per year increases from 39 to 74 (out of 83 IVT-only units in total). At the same time, the number of units with more than 2,000 admissions increases from zero to eleven.

\section{Increasing the number of thrombectomy units}

Our genetic algorithm identified the optimal locations of additional IVT/MT centres, in order to maximise access to MT while also limiting maximum admission numbers to all units (figure 5). As the number of units providing both IVT and MT increases, time to MT reduces and the net clinical benefit of reperfusion treatment increases from 31.1 to 36.2 additional good outcomes per 1,000 admissions with all 107 units providing both IVT and MT. However, beyond about 30 IVT/MT centres, the capacity to maintain 150 procedures per year at each centre markedly reduces. If all IVT/MT centres are required to perform at least $150 \mathrm{MT}$ procedures per year the maximum clinical benefit, achieved with 29 IVT/MT centres, is 31.5 additional disability-free outcomes per 1,000 admissions.

\section{Discussion}

Our modelling study of acute stroke provision in England, and our comparative analysis of the differing configurations of IVT-only units and IVT/MT centres, has produced several key findings of direct relevance to the implementation of reperfusion treatment to maximise the benefits for the population from this highly effective but disruptive innovation. First, we have shown that the population benefit from reperfusion is maximised when the proportion of units that are MT-capable is highest. Put another way, the closer the ultimate national configuration of acute stroke units is to 
a full mothership model, the greater the benefits achievable from reperfusion as a whole. This can be achieved either by reducing the number of IVT-only units, or by increasing the number of IVT/MT centres, or more pragmatically by undertaking a mixture of both. Other considerations will determine which of these is the most deliverable - for the additional IVT/MT centres, significant barriers to the prompt development of new units are capital cost, workforce and time taken. We have also shown that a full mothership model, or any system heavily biased towards a mothership model, produces unsustainably large admission numbers to a relatively small number of IVT/MT centres. The conclusion from these observations is that the principal purpose of retaining IVT-only units, at least from the point of view of reperfusion, is not so much to confer additional benefit from the earlier, more local provision of IVT, but instead to mitigate unsustainable pressures on the IVT/MT centres, some of which would otherwise be several times larger than the current largest stroke unit in England. This illustrates the balance that has to be struck between the benefits to the individual from earlier reperfusion and the dis-benefits to the population from destabilising the acute stroke care system as a whole.

Second, although we have identified that a hybrid model is essential to create a sustainable system, we have also identified some important factors that would destabilise any hybrid system. Principal among these is the natural inclination for ambulance paramedics to travel a little further in order to improve the chances of reperfusion treatment for the individual patient under their care, which we have called 'ambulance bias'. This phenomenon is well recognised anecdotally, with some IVT/MT centres reporting ambulances travelling up to 30 minutes further to deliver their patient to a MTcapable site (C Roffe, personal communication). This has the net effect of converting any hybrid system towards a de facto mothership model, with a pronounced increase in the proportion of patients conveyed directly to an IVT/MT centre, and a corresponding large reduction in admissions to IVT-only units, effectively negating the mitigating effect of having IVT-only units at all. These considerations of institutional viability (at either end of the scale for stroke admissions) have not featured in the reasoning behind other configuration analyses based on additional transport time [25]. In its most extreme form, a model based on ambulances taking the patient directly to the site that offers the patient the best chance of a good outcome would result in $94 \%$ of patients being conveyed directly to IVT/MT centres, and deplete all but 19 IVT-only units in England of any admissions at all.

Third, we have highlighted the limitations of current pre-hospital diagnostics in appropriately selecting patients for conveyance directly to an IVT/MT centre - as in the example of the low specificity of the RACE pre-hospital clinical assessment screening test for LAO [20]. This low specificity has a predictably similar effect to ambulance bias in effectively converting a hybrid model towards a mothership system. The RACE test has been proposed as a means of concentrating the resources of IVT/MT centres on those most likely to benefit, based on pilot data in Catalonia, an area with a population of 7.5M served by 5 MT-capable sites [26]. However, recent unpublished data from the follow-up randomised trial in Catalonia of the use of the RACE test in determining the initial destination of an ambulance conveying a patient with suspected stroke has not shown any disability benefit from such a policy [25]. This finding should therefore prompt caution in the interpretation of studies of pre-hospital diagnostics and in how they might be applied to the NHS in England, with its much lower proportion of patients (27\%) for whom an IVT/MT centre is their nearest acute stroke unit. It is also noteworthy that most diagnostic tests being developed are for LAO, rather than suitability for MT, and so some further selection is required to filter out those 
LAO patients who would benefit from MT. For the time being at least, until other pre-hospital diagnostic tests with superior specificity are developed (whether they be clinical assessment scales, biomarkers or teleconsultation [27]), prehospital selection does not offer the prospect of improving the population benefit from reperfusion without jeopardising the stability of a hybrid system. This is a key area for further service development and research.

Our study does have some limitations. We have based our model on assumptions regarding a reasonably achievable level of both IVT and MT, at 20\% and 10\% of total admitted stroke cases respectively [8]. For MT, the main limitations include initial diagnosis of LAO, angiographic theatre and MT centre capacity, and workforce. For IVT, these levels of treatment have been achieved in many regions and countries elsewhere in Europe $[28,29]$ but there is persisting doubt about whether such levels can be consistently achieved in the UK, and, despite many quality improvement initiatives, the proportion of total patients with a stroke receiving IVT treatment has remained unchanged at $11-12 \%$ over the last 7 years [2]. Not only this, but a five-fold variation between sites in IVT rates persists, an observation that is only partly explained by casemix. The eradication of such a wide degree of variation would be a substantial challenge in any healthcare system and the evidence base for methods for doing so on such a scale is not strong. Nonetheless, any reduction in variation, coupled with any increase in the overall rate of both IVT and MT, would confer a very substantial population benefit even if it falls short of the ambitions of the NHS England Long Term Plan which are used as a basis for the modelling in this study. Furthermore, the observations in our study regarding the relative effects of the different policies are independent of the overall level of reperfusion treatment applied. The twin objectives of increasing reperfusion use and reducing geographical variation both contribute to the overarching aim of significantly increasing the number of patients left disability-free from acute stroke treatments.

The second limitation is that the model has been limited both to the acute aspect of the stroke pathway and to the measurable benefits from reperfusion therapy only. We have not had data on which to base any assumptions about the unintended consequences of the sort of service transformation modelled in this study. In particular, in a mothership or predominantly mothership model, such a policy inevitably involves large numbers of patients being conveyed to an IVT/MT centre for whom MT is either not indicated (e.g. lacunar stroke, cerebral haemorrhage) or inappropriate, for example because of significant co-morbidities. Such patients stand to benefit not at all from being redirected to an IVT/MT centre rather than their local IVT-only unit, and could quite plausibly suffer harm, if only through being displaced from their local health and social services. Relatively small disbenefits, although unmeasured but applied to the large proportion of stroke patients for whom reperfusion is not a viable treatment option (80\%), could quite readily negate much of the more easily recognised benefits from improved access to reperfusion for the minority of patients. Bearing in mind that some of the differences in population benefit between respective configurations can be numerically quite small, these disbenefits could be decisive, but substantiating them has not been feasible in this study.

A further limitation is the extent to which we have been able to model stroke mimic activity. The meaning of a 'stroke mimic' differs according to where the patient is along the pathway of care - it may reflect FAST-positive patients conveyed to hospital by ambulance services on suspicion of stroke, or patients selected in Emergency Departments for admission to a stroke unit [30]. Published data for this latter category would suggest a rule of up to 'one third again' for mimics [31,32], which would substantially inflate the figures we have used for admissions to all sites in our 
modelling. However defined, stroke mimics would consistently increase conveyances and admissions across the board, but they would not affect the relative differences between the various policies if uniformly applied. Thus, they need to be considered when planning for overall levels of emergency activity for ambulance services and acute sites, but they do not influence the discrimination between the various scenarios we have considered.

Another limiting factor to consider is that we have modelled and presented clinical benefit purely in terms of disability-free survival, rather than reductions in other disability states. For instance, converting a patient from severe disability (mRS 4-5) to mild disability (mRS 2) is a significant gain and even reduction from severe to moderate disability (mRS 3 ) is advantageous to individuals and society. We have defined mRS $0-1$ rather than $0-2$ as a good outcome, whereas mRS $0-1$ is in reality an excellent outcome; our modelling therefore presents a conservative picture of the population benefit from the widespread application of reperfusion treatments.

Our estimates of the time-dependent benefits of MT are based on the largest completed study of the effectiveness of MT in a national acute stroke system, the Dutch MR CLEAN randomised controlled trial [33]. We used these data, in preference to the HERMES individual patient metaanalysis [34], on the understanding that MR CLEAN most closely represents the implementation of MT within the English NHS. The HERMES meta-analysis included several studies which used imaging-based selection of later-presenting patients, which has the effect of flattening the curve representing the decay of absolute benefit from MT over time [35]. Currently this category of latepresenting patients represents very few patients treated in England, a situation that is likely to prevail for some time. However, as clinical practice develops with late-presenting and other patients [36,37], this is likely to involve more MT-eligible, IVT-ineligible patients, and thus strengthen the case for a predominantly mothership model. The HERMES meta-analysis reported that $87 \%$ of patients who received MT had first received IVT [34]. However, national stroke audit data for the last three years shows this proportion to be much lower in real-life UK practice at 54\% [2], presumably reflecting the greater use of MT for patients deemed ineligible for IVT who tended to be excluded from the trials. If MT is given to more patients who are ineligible for IVT then the benefit of bypassing an IVT-only unit to be taken directly to an IVT/MT centre will be greater than predicted in our study and further strengthen the case for centralisation.

\section{Conclusions}

Our modelling study of the impact of the implementation of 24/7 MT services across England has identified three key components that should influence policy as the NHS seeks to promote the uptake of this highly effective but disruptive innovation in stroke treatment:

- we have provided reliable estimates of the magnitude of the population benefit from the widespread implementation of reperfusion to the extent anticipated in the NHS England Long Term Plan, with the potential to approximately treble the additional benefit in terms of people with stroke surviving free of disability;

- we have demonstrated how closely linked the full implementation of IVT and MT needs to be if the full extent of the population benefit from reperfusion is to be realised, including the eradication of substantial persisting inter-hospital variation in IVT rates;

- we have modelled several key aspects of pre-hospital management which could destabilise a hybrid system of IVT-only units and IVT/MT centres, along with the areas that require further 
research to advance our understanding of how systems will perform in real-world implementation.

\section{References}

1 Scottish Stroke Care Audit (National Services Scotland). Scottish Stroke Improvement Programme Report 2019.

2019.https://www.strokeaudit.scot.nhs.uk/Publications/docs/2019/Scottish-StrokeImprovement-Programme-2019-National-Report.pdf (accessed 3 Jan 2020).

2 HQIP. Sentinel Stroke National Audit Programme - Annual Report 2019-20. HQIP. 2021.https://www.hqip.org.uk/resource/sentinel-stroke-national-audit-programme-annualreport-2019-20/ (accessed 18 Jan 2021).

3 Patel A, Berdunov V, Quayyum Z, et al. Estimated societal costs of stroke in the UK based on a discrete event simulation. Age Ageing 2019;49. doi:10.1093/ageing/afz162

4 Emberson J, Lees KR, Lyden P, et al. Effect of treatment delay, age, and stroke severity on the effects of intravenous thrombolysis with alteplase for acute ischaemic stroke: A meta-analysis of individual patient data from randomised trials. The Lancet 2014;383:1929-35. doi:10.1016/S0140-6736(14)60583-5

5 Flynn D, Francis R, Halvorsrud K, et al. Intra-arterial mechanical thrombectomy stent retrievers and aspiration devices in the treatment of acute ischaemic stroke : A systematic review and meta-analysis with trial sequential analysis. 2017;2:308-18. doi:10.1177/2396987317719362

6 Sentinel Stroke Audit Programme (SSNAP). SSNAP Apr2018 - Mar2019 Annual Results Portfolio. 2019. https://www.strokeaudit.org/results/Organisational/NationalOrganisational.aspx (accessed 5 Jan 2021).

7 Smith WS, Lev MH, English JD, et al. Significance of large vessel intracranial occlusion causing acute ischemic stroke and tia. Stroke 2009;40:3834-40. doi:10.1161/STROKEAHA.109.561787

8 NHS England. The NHS Long Term Plan. NHS Long Term Plan. 2019. https://www.longtermplan.nhs.uk/publication/nhs-long-term-plan/ (accessed 3 Jul 2019).

9 Allen M, Pearn K, Villeneuve E, et al. Feasibility of a hyper-acute stroke unit model of care across England: a modelling analysis. BMJ Open 2017;7:e018143. doi:10.1136/bmjopen-2017018143

10 Hunter RM, Davie C, Rudd A, et al. Impact on Clinical and Cost Outcomes of a Centralized Approach to Acute Stroke Care in London: A Comparative Effectiveness Before and After Model. PLoS ONE 2013;8:1-9. doi:10.1371/journal.pone.0070420

11 Morris S, Ramsay AIG, Boaden RJ, et al. Impact and sustainability of centralising acute stroke services in English metropolitan areas: retrospective analysis of hospital episode statistics and stroke national audit data. BMJ 2019;364:11. doi:10.1136/bmj.11

12 Wilkinson D. Andrew, Jadhav Ashutosh P., Ducruet Andrew F. Optimizing Next-Generation Care Systems for Stroke. Stroke 2020;51:3190-1. doi:10.1161/STROKEAHA.120.032485

13 McMeekin P, White P, James MA, et al. Estimating the number of UK stroke patients eligible for endovascular thrombectomy Estimating the number of UK stroke patients eligible for endovascular thrombectomy. Eur Stroke J 2017;2:319-26. doi:10.1177/2396987317733343 
14 Bonita R, Beaglehole R. Modification of Rankin Scale: Recovery of motor function after stroke. Stroke J Cereb Circ 1988;19:1497-500.

15 Allen M, Pearn K, Stein K, et al. Estimation of stroke outcomes based on time to thrombolysis and thrombectomy. medRxiv 2020: 2020.07.18.20156653. doi:10.1101/2020.07.18.20156653

16 Holodinsky JK, Williamson TS, Demchuk AM, et al. Drip-and-Ship vs. Mothership: Modelling Stroke Patient Transport for All Suspected Large Vessel Occlusion Patients. JAMA Neurol 2018;75:1477-86. doi:10.1001/jamaneurol.2018.2424

17 Fransen PSS, Beumer D, Berkhemer O a, et al. MR CLEAN, a multicenter randomized clinical trial of endovascular treatment for acute ischemic stroke in the Netherlands: study protocol for a randomized controlled trial. Trials 2014;15:343. doi:10.1186/1745-6215-15-343

18 Froehler MT, Saver JL, Zaidat OO, et al. Interhospital Transfer Before Thrombectomy Is Associated With Delayed Treatment and Worse Outcome in the STRATIS Registry (Systematic Evaluation of Patients Treated With Neurothrombectomy Devices for Acute Ischemic Stroke). Circulation 2017;136:2311-21. doi:10.1161/CIRCULATIONAHA.117.028920

19 Smith Eric E., Kent David M., Bulsara Ketan R., et al. Accuracy of Prediction Instruments for Diagnosing Large Vessel Occlusion in Individuals With Suspected Stroke: A Systematic Review for the 2018 Guidelines for the Early Management of Patients With Acute Ischemic Stroke. Stroke 2018;49:e111-22. doi:10.1161/STR.0000000000000160

20 de la Ossa Herrero N, Carrera D, Gorchs M, et al. Design and Validation of a Prehospital Stroke Scale to Predict Large Arterial Occlusion The Rapid Arterial Occlusion Evaluation Scale. Stroke $J$ Cereb Circ 2013;45. doi:10.1161/STROKEAHA.113.003071

21 Allen M, Pearn K, James M, et al. Maximising access to thrombectomy services for stroke in England: A modelling study. Eur Stroke J 2019;4:39-49. doi:10.1177/2396987318785421

22 Price C, James M. Meeting the Future Challenge of Stroke Meeting the Future Challenge of Stroke. Br. Assoc. Stroke Physicians. 2011.www.basp.ac.uk

23 NHS England. Stroke Services: Configuration Decision Support Guide (www.eoescn.nhs.uk/index.php/download_file/force/2069/168/). 2015.

24 Regarding thrombectomy centre volumes and maximising access to thrombectomy services for stroke in England: A modelling study and mechanical thrombectomy for acute ischaemic stroke: An implementation guide for the UK - PM White, GA Ford, M James, M Allen, 2020. https://journals.sagepub.com/doi/10.1177/2396987320971126 (accessed 18 Dec 2020).

25 Ribo M. Transfer to closest stroke center vs. direct transfer to endovascular stroke center of acute stroke patients with suspected large vessel occlusion in Catalonia (RACECAT): Final results. In: ESO-WSO 2020 Conference Abstracts. 2020. https://eso-wso-conference.org/wpcontent/uploads/sites/42/2020/11/News-from-ESO-WSO-2020-Conference-PR1_Eng.pdf

26 Abilleira S, Tebé C, de la Ossa NP, et al. Geographic dissemination of endovascular stroke thrombectomy in Catalonia within the 2011-2015 period. Eur Stroke J 2017;2:163-70. doi: $10.1177 / 2396987317696376$

27 Mazya MV, Berglund A, Ahmed N, et al. Implementation of a Prehospital Stroke Triage System Using Symptom Severity and Teleconsultation in the Stockholm Stroke Triage Study. JAMA Neurol 2020;77:691-9. doi:10.1001/jamaneurol.2020.0319 
28 Kuhrij LS, Wouters MW, van den Berg-Vos RM, et al. The Dutch Acute Stroke Audit: Benchmarking acute stroke care in the Netherlands. Eur Stroke J 2018;3:361-8. doi:10.1177/2396987318787695

29 Meretoja A, Strbian D, Mustanoja S, et al. Reducing in-hospital delay to 20 minutes in stroke thrombolysis. Neurology 2012;79:306-13. doi:10.1212/WNL.0b013e31825d6011

30 McClelland G, Rodgers H, Flynn D, et al. The frequency, characteristics and aetiology of stroke mimic presentations: a narrative review. Eur J Emerg Med 2019;26:2-8. doi:10.1097/MEJ.0000000000000550

31 Gibson LM, Whiteley W. The differential diagnosis of suspected stroke: a systematic review. $J$ $R$ Coll Physicians Edinb 2013;43:114-8. doi:10.4997/JRCPE.2013.205

32 Dawson A, Cloud GC, Pereira AC, et al. Stroke mimic diagnoses presenting to a hyperacute stroke unit. Clin Med Lond Engl 2016;16:423-6. doi:10.7861/clinmedicine.16-5-423

33 Berkhemer OA, Fransen PSS, Beumer D, et al. A Randomized Trial of Intraarterial Treatment for Acute Ischemic Stroke. N Engl J Med 2015;372:11-20. doi:10.1056/NEJMoa1411587

34 Goyal M, Menon BK, Van Zwam WH, et al. Endovascular thrombectomy after large-vessel ischaemic stroke: A meta-analysis of individual patient data from five randomised trials. The Lancet 2016;387:1723-31. doi:10.1016/S0140-6736(16)00163-X

35 Saver JL, Goyal M, van der Lugt A, et al. Time to Treatment With Endovascular Thrombectomy and Outcomes From Ischemic Stroke: A Meta-analysis. Jama 2016;316:1279. doi:10.1001/jama.2016.13647

36 Nogueira RG, Jadhav AP, Haussen DC, et al. Thrombectomy 6 to 24 Hours after Stroke with a Mismatch between Deficit and Infarct. N Engl J Med Published Online First: 2017. doi:10.1056/NEJMoa1706442

37 Albers GW, Marks MP, Kemp S, et al. Thrombectomy for Stroke at 6 to 16 Hours with Selection by Perfusion Imaging. $N$ Engl J Med 2018;378:708-18. doi:10.1056/NEJMoa1713973 


\section{Figures}

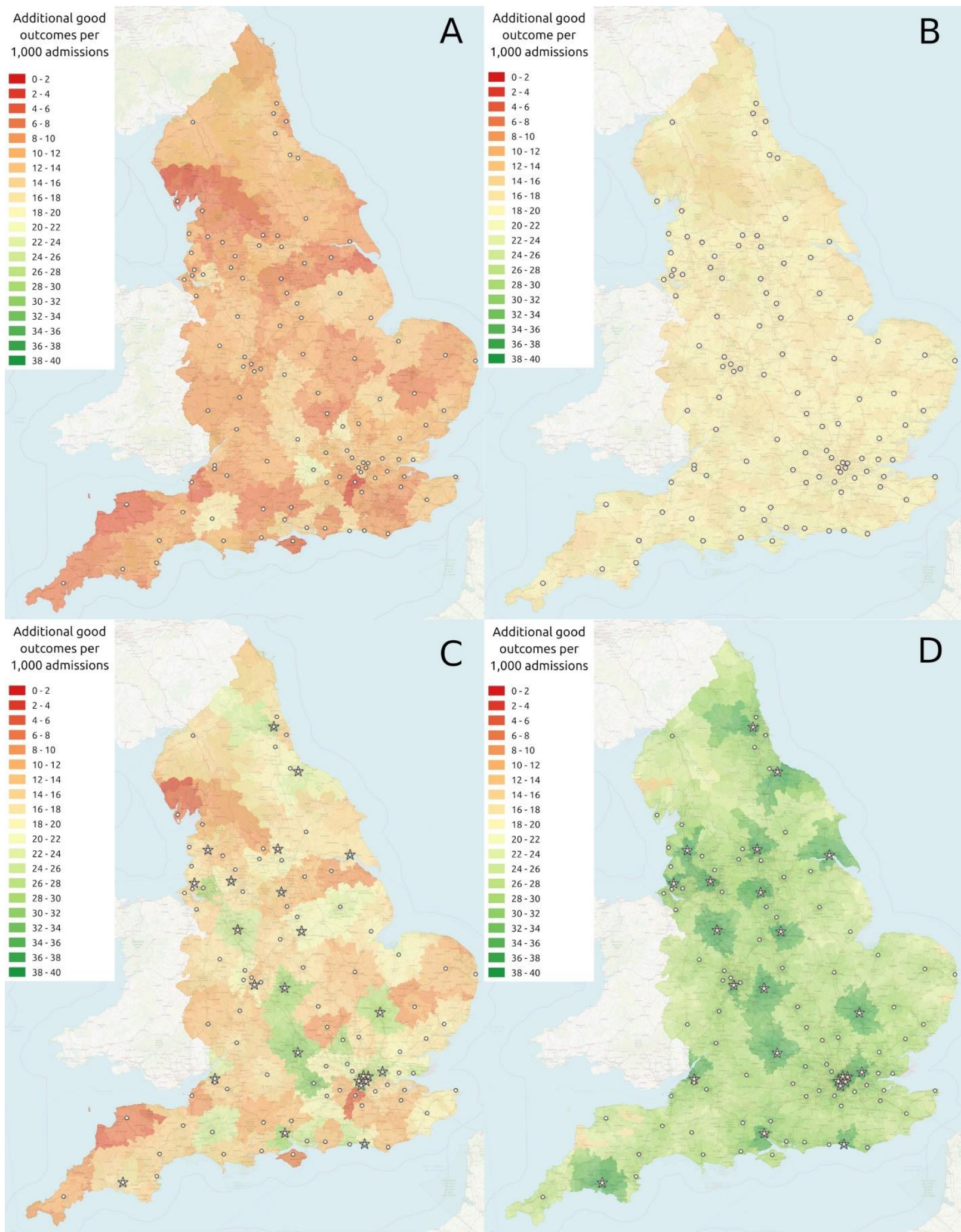

Figure 1: Geographical variation in clinical benefit (additional good outcomes / 1,000 admissions). A) IVT provided at 107 stroke units, with IVT rates for units taken from 2018-19 SSNAP report. B) IVT provided at 107 stroke units, with 20\% IVT at all units. C) IVT provided at 107 stroke units, and MT also provided at the 24 neuroscience centres, with IVT rates for units taken from 2018-19 SSNAP report. D) IVT provided at 107 stroke units, and MT also provided at the 24 neuroscience centres, with 20\% IVT at all units. Circles show IVT-only units, stars show IVT/MT centres, with $10 \%$ patients receiving $M T$. 


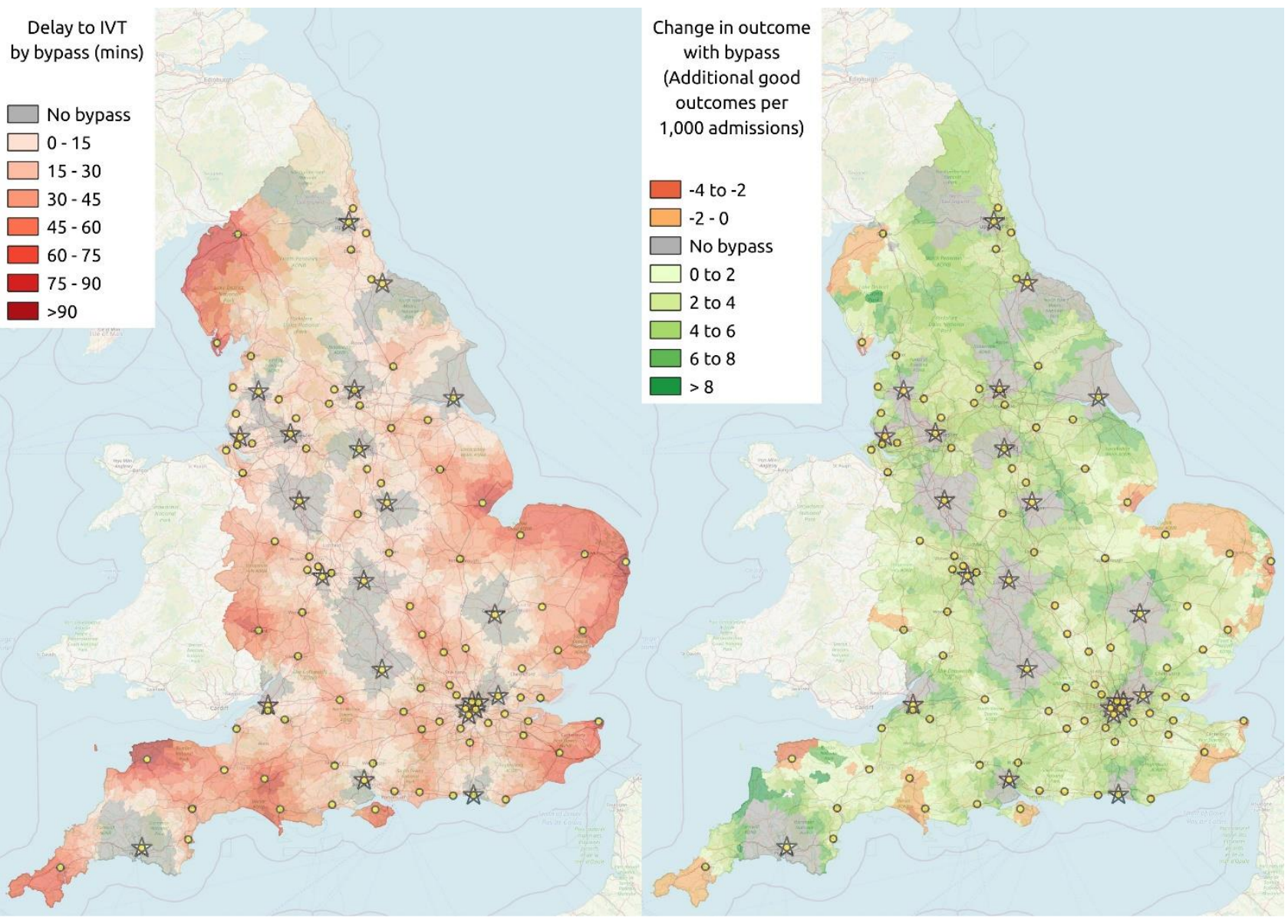

Figure 2: The effect of implementing the mothership model, where all patients bypass their more local IVT-only unit (circles) to directly attend one of the 24 IVT/MT centres (stars). Maps show delay in receiving IVT (left panel) and net change in the clinical benefit of reperfusion (right panel). 


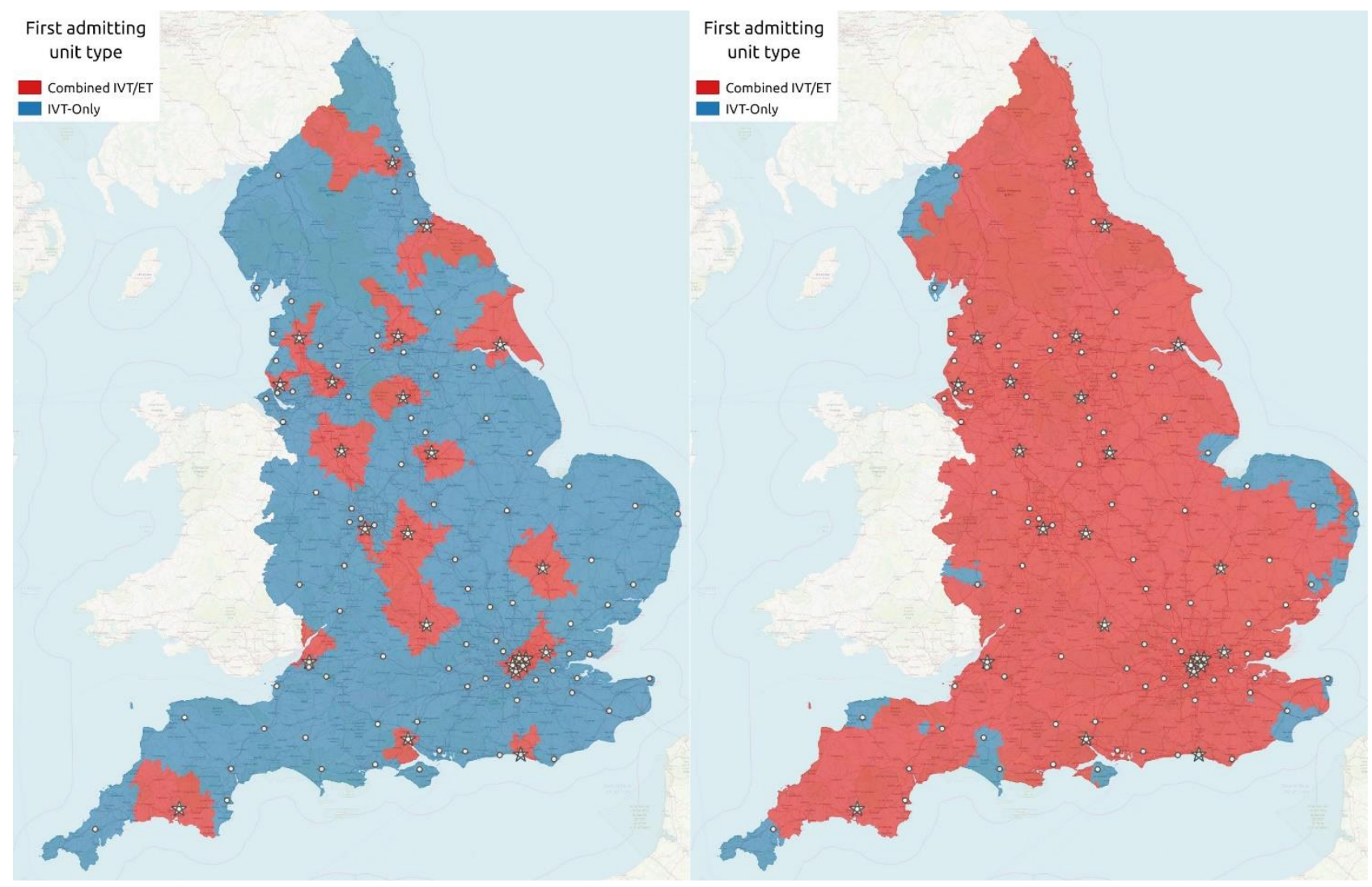

Figure 3: Catchment areas for either IVT/MT centres (stars) or IVT-only units (circles) if decision on conveyance to first stroke unit is made by either closest unit (left panel) or the destination that gives the highest probability of a good outcome (without any pre-hospital diagnostic; right panel). The model has 24 IVT/MT centres at the current neuroscience centres and 83 IVT-only units at the remaining current acute stroke centres. 

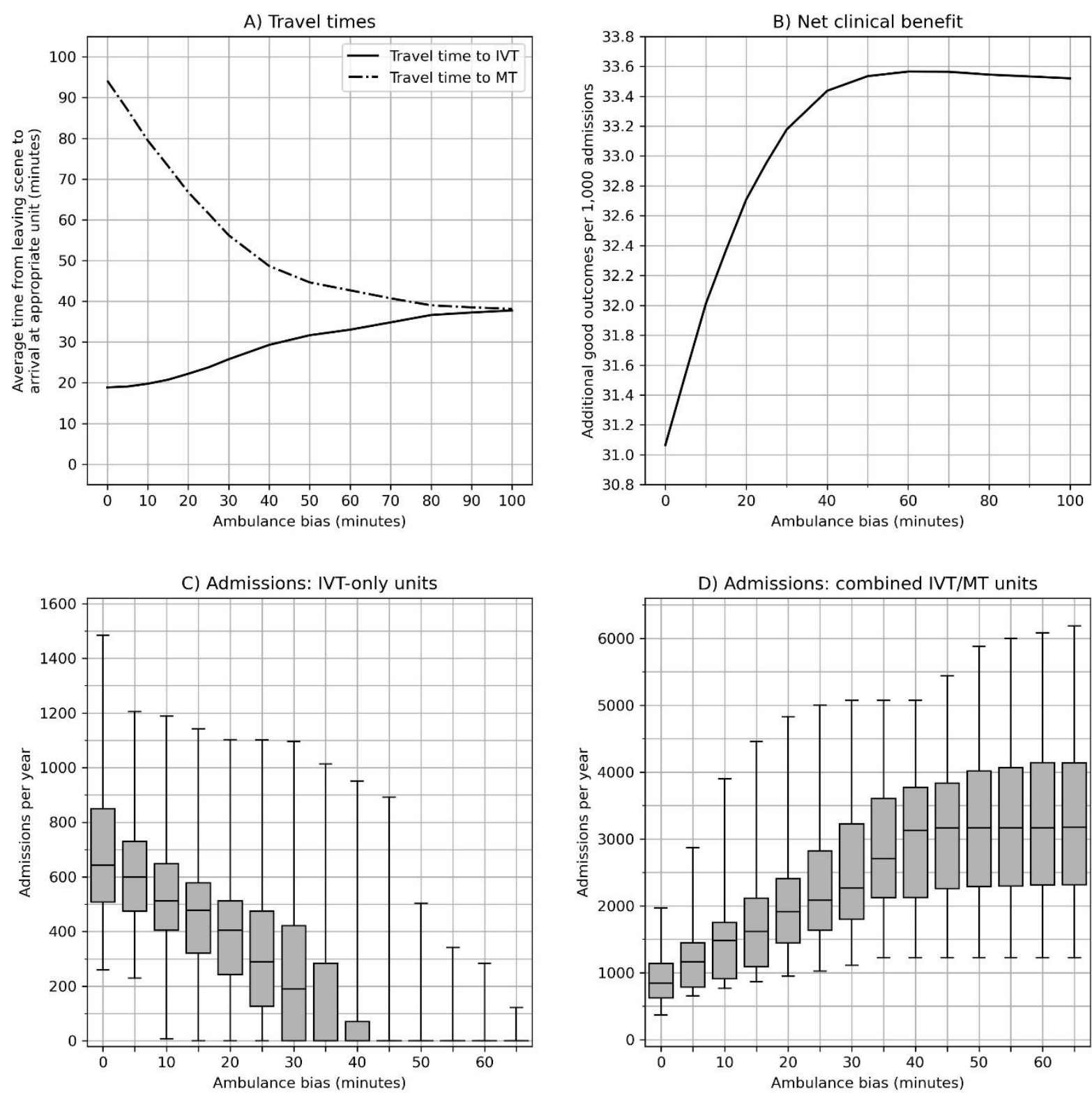

Figure 4: The effect of ambulance bias on travel time to IVT and MT (top left panel), clinical outcome (top right panel), admission numbers to IVT-only units (bottom left panel) and admission numbers to combined IVT/MT centres (bottom left panel). Ambulance bias is measured as the additional travel time an ambulance crew will allow to go directly to an IVT/MT centre instead of a closer IVT-only unit. Boxplots show median (mid-way line), interquartile range (box), range (whiskers) for individual units. If a patient first attends an IVT-only unit, time to the IVT/MT centre will include road transfer time and process related delays. The model has 24 IVT/MT centres at the current neuroscience centres and 83 IVT-only units at the remaining current acute stroke centres. 

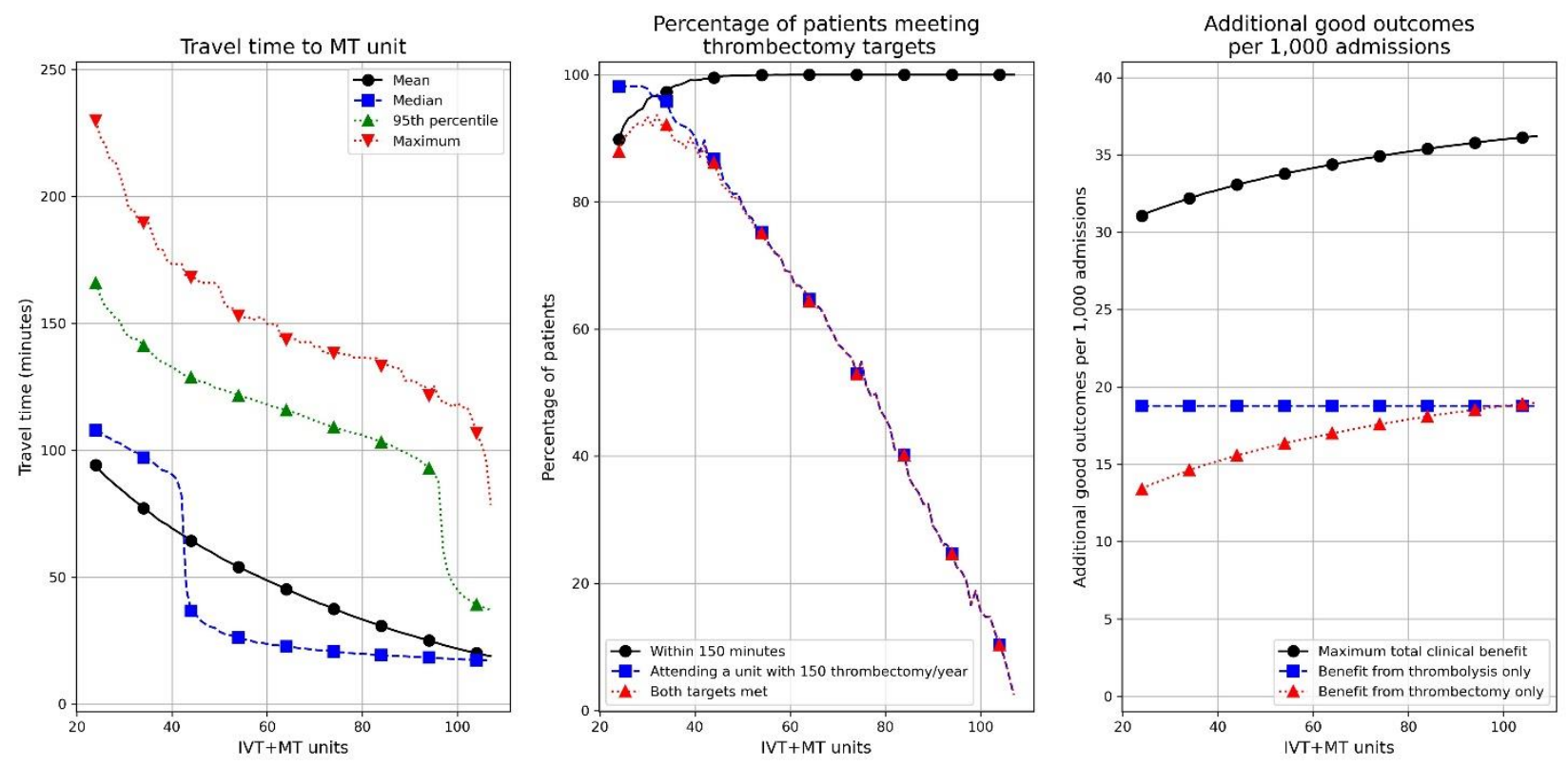

Figure 5: The effect of converting IVT-only units to IVT/MT centres. The charts show effect on travel time to an IVT/MT centre (left panel), the percentage of patients meeting MT admission targets (middle panel), and the clinical benefit of reperfusion (right panel). The base case model has 24 IVT/MT centres at the current neuroscience centres and 83 IVT-only units at the remaining current acute stroke centres. 


\section{Contact}

For queries contactm.allen@exeter.ac.uk

\section{Contribution}

All authors were involved in the design and planning of the work. MA \& KP performed the required coding for the work; all authors reviewed outputs from the work and suggested next steps. All authors have been involved in the writing of the paper.

Ken Stein is the guarantor of the work.

\section{Competing interests}

We have read and understood BMJ policy on declaration of interests and declare the following interests:

Prof. White reports grants from Microvention Terumo, grants from Stryker, grants from Codman, grants from Medtronic, grants from Penumbra, personal fees from Microvention Terumo, outside the submitted work.

GFs organisation has received an unrestricted medical education grant from Medtronic to support development of an implementation guide for mechanical thrombectomy. GF has received personal remuneration from Medtronic and Stryker for advisory board and educational meeting lectures.

\section{Ethics approval}

As this modelling study uses Hospital Episodes Statistics aggregate patient counts by geographical area, no ethical approval is required.

\section{Transparency}

Prof. Martin James affirms that this manuscript is an honest, accurate, and transparent account of the study being reported; that no important aspects of the study have been omitted; and that any discrepancies from the study as planned (and, if relevant, registered) have been explained.

\section{Funding}

The work described was jointly funded by NHS England and National Institute for Health Research (NIHR) Applied Research Collaboration South West Peninsula (SWPenARC).

\section{Disclaimer}

The views and opinions expressed in this paper are those of the authors, and not necessarily those of the NHS, the National Institute for Health Research, or the Department of Health.

\section{Patient and public involvement}

Patients and/or the public were not involved in the design, or conduct, or reporting, or dissemination plans of this research.

\section{Data sharing}

Admissions per lower super output area (LSOA), travel times from all LSOAs to all acute stroke units, and base code used for this model may be found at: https://github.com/MichaelAllen1966/stroke_unit_location 